\title{
Fibromatosis colli in a Neonate - A Rare Case Report
}

\author{
Narendar Reddy .P' ${ }^{1}$, Sravan Kumar .K², Anil U. Madurwar ${ }^{3}$ \\ ${ }^{1}$ Assistant Professor, Department of Radiology, CAIMS, Karimnagar, India \\ ${ }^{2}$ Postgraduate student, Department of Radiology, CAIMS, Karimnagar, India \\ ${ }^{3}$ HOD \& Professor, Department of Radiology, CAIMS, Karimnagar, India
}

\begin{abstract}
Fibromatosis colli or pseudotumor of infancy of the sternocleidomastoid muscle is a rare cause of a benign neck mass in neonates and infants. Though exact etiology is not known, it is most likely due to birth trauma. Ultrasonography is the imaging modality of choice and nearly all the cases are correctly diagnosed if clinical and radiologically suspected. This entity is a self-limiting condition and resolves over a period of time. If diagnosed correctly can be managed conservatively and prevent unnecessary diagnostic and therapeutic maneuvers. We report a rare case of fibromatosis colli in a 1 month old neonate diagnosed using ultrasonography.
\end{abstract}

Keywords: Fibromatosis colli, neonate, pseudotumor, sternocleidomastoid muscle, Ultrasonography.

\section{Introduction}

Fibromatosis colli or pseudotumor of infancy of the sternocleidomastoid muscle is a rare benign cause of neck swelling or mass in neonate and infants. Though the exact etiology is not known, it is most likely due to birth trauma or malposition in uterus. It is one of the cause of congenital torticollis and usually recognized by mother as neck swelling with or without restricted neck movement. Ultrasonography of neck is the imaging modality of choice and sometimes CT scan or MRI scan may be required to further characterize the disease and extent of involvement. Real time ultrasonography demonstrate synchronous motion of the mass with the sternocleidomastoid muscle, thus confirming the diagnosis.

We present a case report of fibromatosis colli in a 1 month old neonate diagnosed using ultrasonography. In addition to this brief discussion was made on literature review, clinical and radiological findings and differentials.

\section{Case Report}

A one month old neonate was referred to the radiology department for ultrasonography of a neck swelling on the right side which was incidentally noticed by the mother 10 days ago. On examination the neck swelling was firm, freely mobile, no warm on touch and approximately $1.5-2 \mathrm{~cm}$ soft tissue mass was felt on the right side of neck (figure 1). Neonate was afebrile. There was restriction of neck movements on the affected side and chin is directed away. Past history revealed prolonged labour with breech presentation; however no assisted delivery was noted. Baby did not cry immediately after birth and was admitted for three days in neonatal intensive care unit. Later baby started feeding well and was immunized till date. Ultrasonography of neck showed ill-defined fusiform thickening of right distal sternocleidomastoid muscle with heterogeneous echotexture (figure 2). The fibrillar structures of muscle fibers are well maintained. On color doppler study, minimal peripheral vascularity with high resistance flow is noted (figure 3 ). No significant cervical nodes or vascular engorgement was noted. Left sternocleidomastoid muscle was normal in bulk and signal intensity. In view of clinical history and ultrasonography findings a diagnosis of fibromatosis colli or pseudotumor of the sternocleidomastoid muscle was considered. The condition was managed conservatively. Physiotherapy and neck stretching exercises were started.

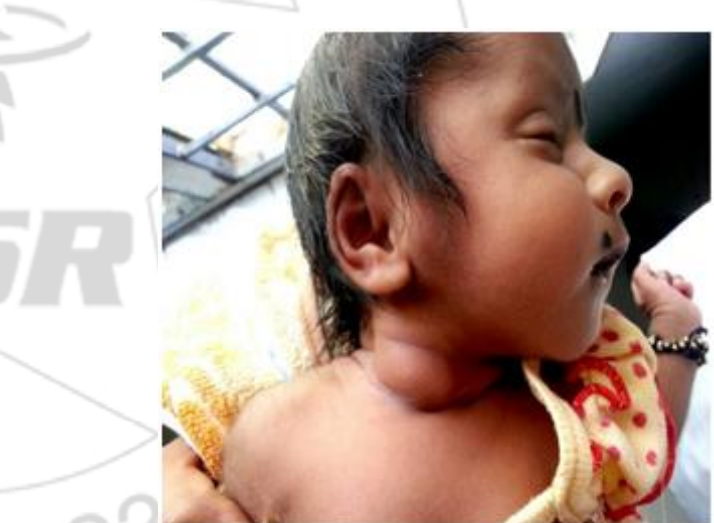

Figure 1: A one month old neonate showing a neck swelling on right side

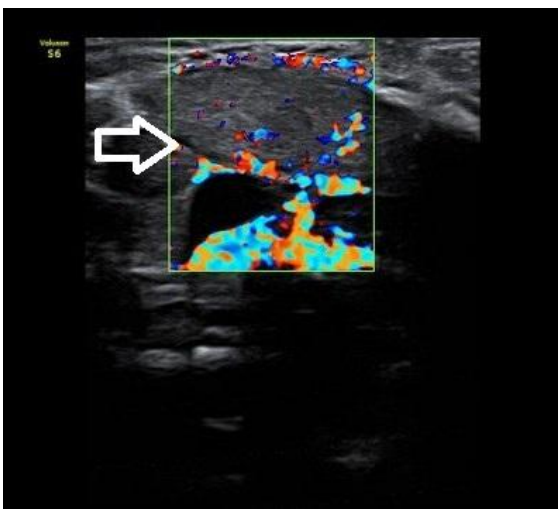

Figure 2: Longitudinal USG image of the neck show fusiform enlargement of the distal right sternocleidomastoid muscle (right) 


\section{International Journal of Science and Research (IJSR) \\ ISSN (Online): 2319-7064}

Index Copernicus Value (2013): 6.14 | Impact Factor (2015): 6.391

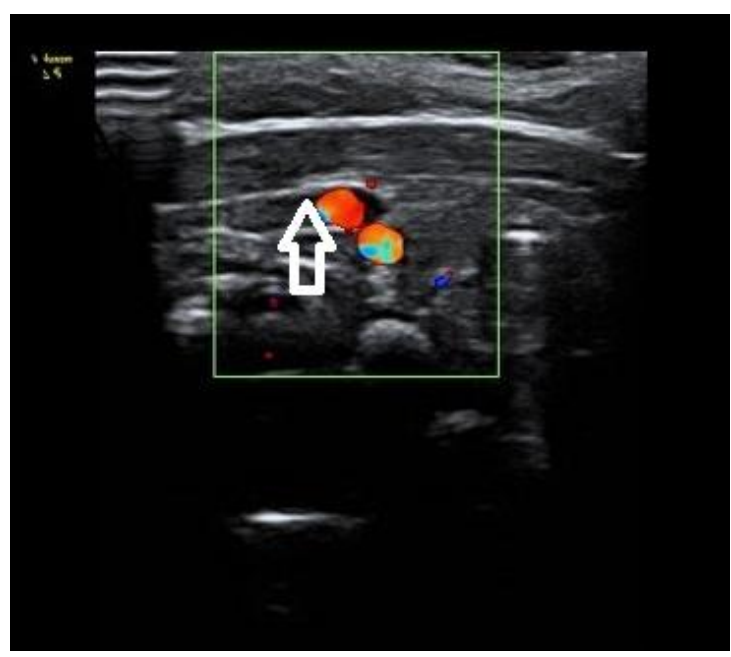

Figure 3: Longitudinal USG image of normal sternocleidomastoid muscle (left).

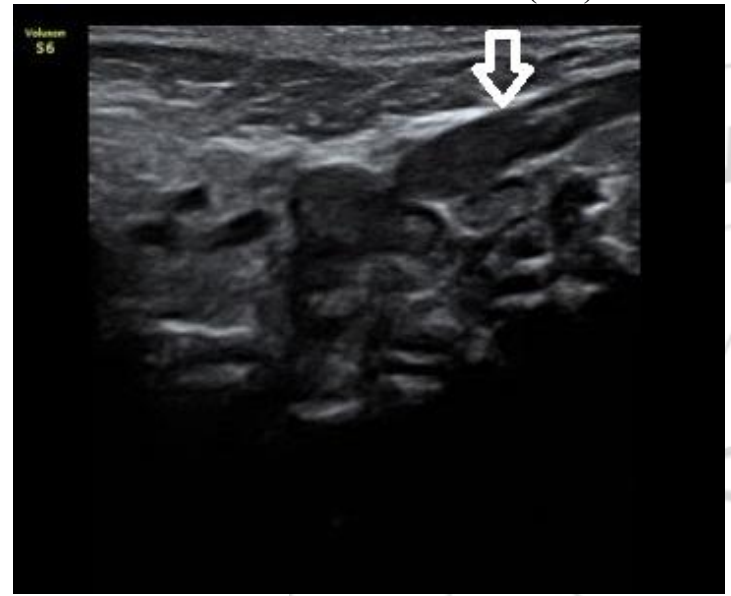

Figure 4: Transverse USG image of the neck shows oval soft tissue mass at distal right sternocleidomastoid muscle

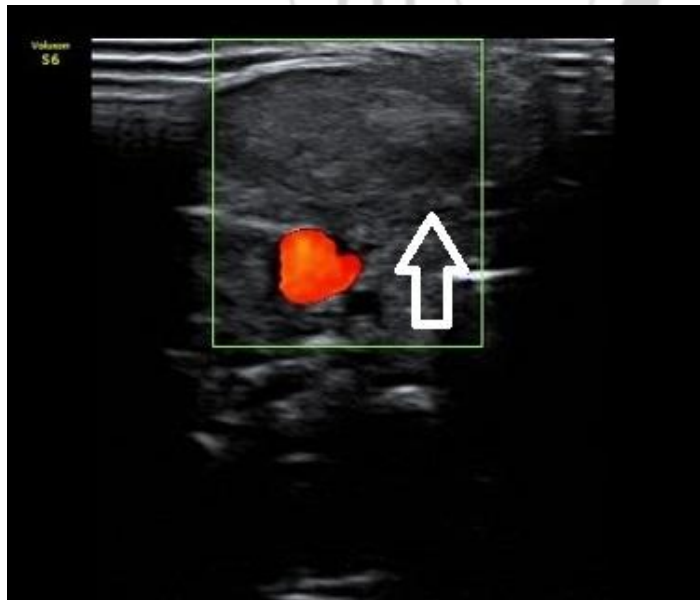

Figure 5: Transverse USG image with normal bulk in the left sternocleidomastoid muscle

\section{Discussion}

Fibromatosis colli is a rare benign condition in neonates and infants with neck swelling. Most cases show no abnormality at birth but manifest between the 2nd and 4th weeks of life as a firm soft-tissue mass in the lower one-third of the sternocleidomastoid muscle1, 6. It is usually unilateral, slightly more common on the right side while bilateral involvement is rare. It affects both the sternal and clavicular heads of the muscle and seen more commonly in male child1. About 14-20\% cases of congenital torticollis are due to contraction of the sternocleidomastoid muscle2. The exact cause is unclear; it is likely related to birth trauma greater than $90 \%$ of cases associated with a difficult or forceps delivery. Some cases are due to abnormal utero fetal head position, which causes selective injury and leads to development of a secondary compartment syndrome, pressure necrosis and fibrosis within the sternocleidomastoid muscle2 . No cervical lymphadenopathy, vascular invasion or bony involvements are seen as compared other neck masses.

Ultrasonography (USG) is the best imaging modality for diagnosis due to its relative low cost, its lack of radiation and the proximity of the lesion to the skin 4. USG findings show fusiform thickening or diffusely enlarged sternocleidomastoid muscle with variable echogenicity. Color doppler interrogation may reveal a high resistance waveform. The enlarged area often moves synchronously with the rest of the sternocleidomastoid muscle (SCM) on real time sonography 4. Almost all the cases can be identified by ultrasonography as compared to $\mathrm{CT}$ or MRI. Computed tomography (CT) show a homogeneous diffusely enlarged sternocleidomastoid muscle with surrounding well preserved fat planes. At times calcification may be present5. Magnetic resonance imaging (MRI) shows decreased signal intensity of the mass on T2W images as compared to gradient-recalled $\mathrm{T} 1 \mathrm{~W}$ images, because of the presence of fibrous tissue7. The extent of involved muscle is better delineated with MRI than with USG. Radiographs are usually normal and sometimes used to exclude congenital bony abnormality. Histological features include blandappearing fibroblasts, myofibroblasts and atrophic skeletal muscle along with muscle giant cells and bare nuclei 8 .

Fibromatosis colli is self-limiting condition and usually resolves within 4-8 months and requires physiotherapy and neck stretching exercises 9 . Approximately two-third cases regress by the age of 2 years 3 . The differential diagnoses of solid masses in the neck include neuroblastoma, rhabdomyosarcoma, lymphoma and other sarcomas. In addition to neck mass, may have enlarged cervical lymph nodes, vascular encasement, or invasion of surrounding structures. Cystic mass in the neck near the midline includes cystic hygroma, branchial cleft cyst, dermoid cyst, teratoma or thyroglossal duct cyst are considered in the differentials.

\section{Conclusion}

Our case highlights, fibromatosis colli or pseudotumor of infancy of the sternocleidomastoid muscle is a rare benign cause of neck swelling or mass in neonate and infants. The radiologist must be aware of its imaging features in order to differentiate it from other neck masses. This entity is a selflimiting condition and resolves over a period of time. If diagnosed correctly can be managed conservatively and prevent unnecessary diagnostic and therapeutic maneuvers.

\section{Acknowledgements}

The authors reported no conflict of interest and no funding 
was received in this work.

\section{References}

[1] Enzinger FM, Weiss SW. Soft tissue tumors 3rd ed. St Louis, Mo: Mosby, 1995.

[2] Davids JR, Wenger DR, Mubarak SJ. Congenital muscular torticollis: sequelae of intrauterine or perinatal compartment syndrome. J Pediatr Orthop 1993; 13:141 147.

[3] Coventry MB, Harris LE, Bianco AJ, Bulbulian AH. Congenital muscular torticollis (wryneck). Postgrad Med 1960; 28:383-391.

[4] Patrick LE, O'shea P, Simoneaux SF et-al. Fibromatoses of childhood: the spectrum of radiographic findings. AJR Am J Roentgenol. 1996;166 (1): 163-9.

[5] Meuwly JY, Lepori D, Theumann N et-al. Multimodality imaging evaluation of the pediatric neck: techniques and spectrum of findings. Radiographics. 25 (4): 931-48.

[6] Robbin MR, Murphey MD, Temple HT et-al. Imaging of musculoskeletal fibromatosis. Radiographics. 21 (3): 585-600.

[7] Ablin DS, Jain K, Howell L, Steel D. West-Ultrasound and MR imaging of Fibromatosis Colli Pediatr Radiol. 1998; 28:230-3.

[8] Sharma S, Mishra K, Khanna G. Fibromatosis colli in infants- a cytologic study of eight cases. Acta Cytol. 2003; 47:359-62.

[9] Crawford SC, Harnsberger HR, Johnson L et-al. Fibromatosis colli of infancy: CT and sonographic findings. AJR Am J Roentgenol. 1988;151 (6): 11834. 